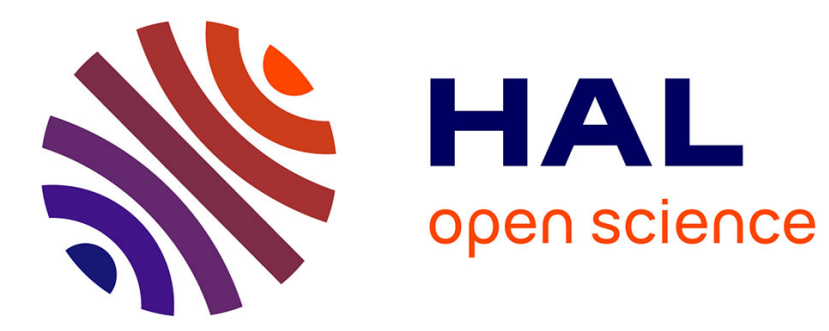

\title{
Best Basis Denoising with Non-stationary Wavelet Packets
}

\author{
Nizar Ouarti, Gabriel Peyré
}

\section{To cite this version:}

Nizar Ouarti, Gabriel Peyré. Best Basis Denoising with Non-stationary Wavelet Packets. ICIP'09, Nov 2009, Cairo, Egypt. pp.3825-3828, 10.1109/ICIP.2009.5414264 · hal-00410869

\section{HAL Id: hal-00410869 \\ https://hal.science/hal-00410869}

Submitted on 24 Aug 2009

HAL is a multi-disciplinary open access archive for the deposit and dissemination of scientific research documents, whether they are published or not. The documents may come from teaching and research institutions in France or abroad, or from public or private research centers.
L'archive ouverte pluridisciplinaire HAL, est destinée au dépôt et à la diffusion de documents scientifiques de niveau recherche, publiés ou non, émanant des établissements d'enseignement et de recherche français ou étrangers, des laboratoires publics ou privés. 


\section{BEST BASIS DENOISING WITH NON-STATIONARY WAVELET PACKETS}

\author{
Nizar Ouarti \\ INRIA \\ nizar.ouarti@irisa.fr
}

\author{
Gabriel Peyré \\ Université Paris-Dauphine \\ gabriel.peyre@ceremade.dauphine.fr
}

\begin{abstract}
This article introduces a best basis search algorithm in a nonstationary (NS) wavelet packets dictionary. It computes an optimized labeled quad-tree that indexes the filters used for the NS wavelet packets decomposition. This algorithm extends the classical best basis search by exploring in a hierarchical manner the set of NS wavelet packets coefficients. The scale-by-scale variation of the filters adapts the transform to the frequency content of complex textures. The resulting denoising method is made translation invariant by cycle spinning. Numerical results show that NS wavelet packets give better results than wavelet packets and waveatoms for the denoising of natural images, in particular in textured areas. Moreover, the cycle spinning method increases significantly the denoising abilities of our algorithm ${ }^{1}$.
\end{abstract}

Index Terms - wavelet, wavelet packet, best basis, denoising

\section{INTRODUCTION}

Fixed basis denoising. Wavelet bases capture efficiently transient parts of signals and images. In particular, orthogonal wavelet bases are optimal for the approximation and denoising of piecewise smooth signals and images with bounded variations [1].

Natural images contain complex structures such as regular edges and oscillating textures. In the image setting, wavelets are sub-optimal to represent both textures and edges. Elongated oriented atoms such as curvelets [2] should be used to capture efficiently cartoon edges. Locally oscillating atoms such as local DCT [1], brushlets [3] or waveatoms [4] should be preferred to process oscillating oriented textures,.

Best basis denoising. Each of these fixed bases are well suited to process a specific kind of structures in images. A best basis algorithm computes an adapted orthogonal basis within a structured dictionary. This best basis selection is applied to a noisy image to perform an adaptive denoising. This adaptive processing can alleviate some of the difficulties that faces a fixed representation. For instance, a best wavelet

\footnotetext{
${ }^{1}$ This work is supported by ANR grant NatImages ANR-08-EMER-009.
}

packet denoising [5] is useful to denoise oscillating textures such as fingerprints by performing an adapted segmentation of the frequency domain.

Contribution. This paper extends the best wavelet packet adaptivity for image denoising by using non-stationary (NS) wavelet packets introduced by Cohen and Séré [6]. We show that the set of all bases is parameterized by a set of labeled quadtrees. We propose a new best basis selection method that extends classical dynamical programming method to the non-stationary setting. This algorithm shares similarities with the search in multi-tree dictionaries [7]. A translation invariant extension of the best NS wavelet packets thresholding further enhances the denoising quality by reducing denoising artifacts. Numerical results show that the resulting adaptive NS denoising method improves over wavelet packets and waveatoms denoising over textured areas.

\section{NON-STATIONARY WAVELET PACKETS}

Quadtree parameterization. A 2D NS wavelet packet basis $\mathcal{B}(\lambda)$ is parameterized by a quad-tree $\lambda$. The nodes $(j, i)$ of $\lambda$ are indexed by a scale $0 \leqslant j \leqslant J=\log _{2}(n) / 2$ representing the depth in the tree, and a position $0 \leqslant i<4^{j}$.

Each node $(j, i)$ is assigned a label $\lambda_{j, i} \in\{0, \ldots, S-$ $1\} \cup \varnothing$. A leaf node is such that $\lambda_{j, i}=\varnothing$, and it has no child node. We denote by $\mathcal{L}(\lambda)$ the set of leaves. An interior node $(j, i)$ is such that $\lambda_{j, i} \in\{0, \ldots, S-1\}$ and it has 4 children nodes indexed as $(j+1,4 i), \ldots,(j+1,4 i+3)$. We denote by $\mathcal{I}(\lambda)$ the set of interior nodes. Figure 1 shows an example of such a quadtree $\lambda$.

Wavelet filters. Each label $\lambda_{j, i}$ indexes a 1D low pass filter chosen in a set $\left\{h_{\ell}\right\}_{\ell=0}^{S-1}$. For each index $\ell$, the corresponding high pass quadrature filter is $g_{\ell}$, and 2D orthogonal tensorial filters $\left\{h_{\ell}^{\eta}\right\}_{\eta=0}^{3}$ are computed as

$$
h_{\ell}^{0}=h_{\ell} \otimes h_{\ell}, h_{\ell}^{1}=h_{\ell} \otimes g_{\ell}, h_{\ell}^{2}=g_{\ell} \otimes h_{\ell}, h_{\ell}^{3}=g_{\ell} \otimes g_{\ell} .
$$

NS wavelet packet transform. The forward NS wavelet packet transform computes coefficients

$$
\mathcal{W}_{\lambda}(f)=\left\{f_{j, i}\right\}_{(j, i) \in \mathcal{L}(\lambda)}
$$




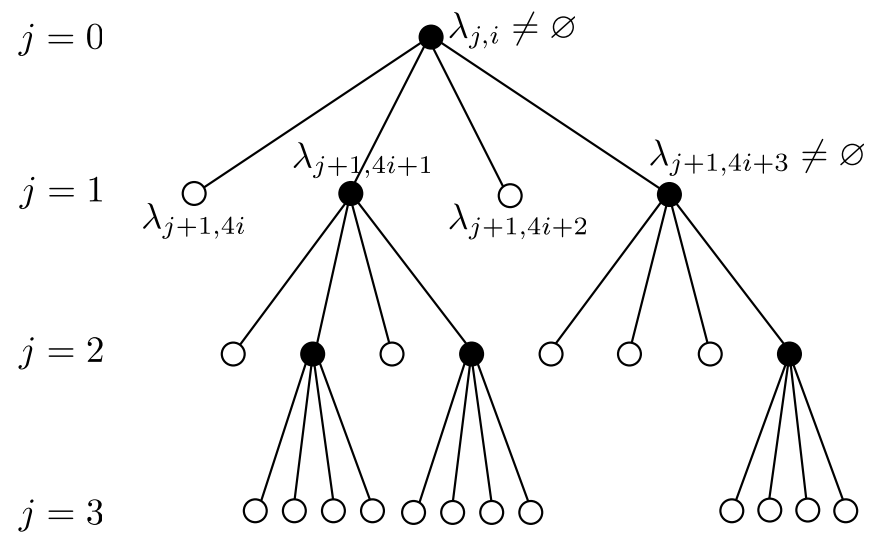

Fig. 1. Example of quadtree $\lambda$ that defines a $2 D N S$ wavelet packet basis $\mathcal{B}(\lambda)$.

of a discrete image $f \in \mathbb{R}^{n}$ of $n$ pixels, that are stored on the leaves $\mathcal{L}(\lambda)$ of the tree $\lambda$. The computation is performed with an iterative algorithm that follows the edges of the tree $\lambda$. It starts at scale $j=0$, where $f_{0,0}=f$. For each scale $0<j<J=\log _{2}(n) / 2$, for each interior node $(j, i) \in \mathcal{I}(\lambda)$, the signals on the children nodes is defined as

$$
\forall 0 \leqslant \eta<4, \quad f_{j+1,4 i+\eta}=\left(f_{j, i} * h_{\ell}^{\eta}\right) \downarrow 2
$$

where $\ell=\lambda_{j, i}$ is the index of the filter, and $\downarrow 2$ is the operator that subsamples an image by a factor 2 along each direction.

The backward NS wavelet packet transforms retrieves an image $f=\mathcal{W}_{\lambda}^{*}(F) \in \mathbb{R}^{n}$ from a set of coefficients $F=$ $\left\{f_{j, i}\right\}_{(j, i) \in \mathcal{L}(\lambda)}$. For each scale $J>j>0$, the signal on the interior node $(j, i) \in \mathcal{I}(\lambda)$ is recovered as

$$
f_{j, i}=\sum_{\eta=0}^{3}\left(f_{j+1,4 i+\eta} \uparrow 2\right) * \tilde{h}_{\ell}^{\eta}
$$

where $\tilde{h}[n]=h[-n]$ and $\uparrow 2$ is the upsampling operator that inserts a zero at each odd location along each direction. The image is recovered on the root node as $f=f_{0,0}$. Both the forward and the backward transform are computed in $O\left(n \log _{2}(n)\right)$ operations.

\section{BEST NS WAVELET PACKET BASIS}

Processing an image $f \in \mathbb{R}^{n}$ is performed by modifying the coefficients of $f$ in an optimized NS wavelet packet basis $\mathcal{B}\left(\lambda^{\star}\right)$. The quadtree $\lambda^{\star}$ is selected by minimizing a Lagrangian

$$
L(f, \mathcal{B}(\lambda))=\sum_{(j, i) \in \mathcal{L}(\lambda)}\left(\rho+\sum_{m=0}^{n / 4^{j}-1} \Phi\left(f_{j, i}[m]\right)\right)
$$

where $\left\{f_{j, i}\right\}_{(j, i) \in \mathcal{L}(\lambda)}=\mathcal{W}_{\lambda}(f)$ and where $\Phi: \mathbb{R} \rightarrow \mathbb{R}$ is a cost function that depends on the application (denoising, compression, ...). The parameter $\rho \geqslant 0$ is a penalization that reduces the complexity of the best basis tree.

The tree $\lambda^{\star}$ adapted to an image $f$ is computed by exploiting the hierarchical structure of the wavelet packet coefficients.

Step 1 - Computing all the coefficients. The set of all possible NS wavelet packets coefficients is obtained by a top to bottom filtering process. The coefficients on the root node are $F_{0,0}=f$. For each scale $0<j<J$, for each index $0 \leqslant k<(4 S)^{j}$, the coefficients on the $4 S$ children nodes of $F_{j, i}$ are obtained by computing all possible filterings

$\forall 0 \leqslant \ell<S, \forall 0 \leqslant \eta<4, F_{j+1,4 S k+4 \ell+\eta}=\left(F_{j, i} * h_{\ell}^{\eta}\right) \downarrow 2$.

Step 2 - best filters selection. A bottom to top recursive selection process selects the best filters for each scale $j$ and index $0 \leqslant k<(4 S)^{j}$. The Lagrangian is evaluated for each index $k$ at the finest scale $j=J$

$$
\forall 0 \leqslant k<(4 S)^{J}, L_{J, k}=\sum_{m=0}^{n / 4^{J}-1} \Phi\left(F_{J, i}[m]\right) .
$$

For each $J<j \leqslant 0$, the cumulated Lagrangian is computed for each application of a filter indexed by $0 \leqslant \ell<S$

$$
L_{j, k}^{\ell}=\rho+\sum_{\eta=0}^{3} L_{j+1,4 S k+4 \ell+\eta} .
$$

The best filter index defines the Lagrangian at the next scale for each $0 \leqslant k<(4 S)^{j}$ that minimizes the cumulative Lagrangian

$$
L_{j, k}=\min \left(\min _{0 \leqslant \ell<S} L_{j, k}^{\ell}, \sum_{m=0}^{n / 4^{j}} \Phi\left(F_{j, k}[m]\right)\right) .
$$

If $L_{j, k}=L_{j, k}^{\ell}$ for some $0 \leqslant \ell<S$, the best filter choice is set to $\ell_{j, k}=\ell$, otherwise, the filtering is stopped and $\ell_{j, k}=\varnothing$.

Step 3 - Best tree construction. Once the best filter choices $\ell_{j, k}$ are computed for all $j$ and all $0 \leqslant k<(4 S)^{j}$, the best tree $\lambda^{\star}$ with indexes $\lambda_{j, i}^{\star}$ is computed for all $j$ and all $0 \leqslant$ $i<4^{j}$. The initial tree is initialized with a single root node $\mathcal{L}\left(\lambda^{\star}\right)=\{(0,0)\}$, together with a filter choice $\lambda_{0,0}^{\star}=\ell_{0,0}$ and with a link $\pi_{0,0}=0$.

For each $0 \leqslant j<J$, for each node $(j, i)$ such that $\ell=$ $\lambda_{j, i}^{\star} \neq \varnothing$, we retrieve the link $k=\pi_{j, i}$, and add the children nodes to the leaves of the optimal tree $\lambda^{\star}$,

$$
\mathcal{L}\left(\lambda^{\star}\right) \leftarrow \mathcal{L}\left(\lambda^{\star}\right) \cup\{(j+1,4 i+\eta)\}_{\eta=0}^{3} .
$$

The label for each new node is set to

$$
\forall 0 \leqslant \eta<4, \quad \lambda_{j+1,4 i+\eta}=\ell_{j+1,4 S k+4 \ell+\eta}
$$

and the link is defined as $\pi_{j+1,4 i+\eta}=4 S k+4 \ell+\eta$. 
Complexity. The numerical complexity of this best basis algorithm is dominated by the computation of the set of coefficients $\left\{F_{j, k}\right\}_{(j, k)}$ for all possible $0 \leqslant j<J=\log _{2}(n) / 2$ and $0 \leqslant k<(4 S)^{j}$. For each scale $j=1, \ldots, J$, one needs to compute $(4 S)^{j}$ filterings of vectors of size $n / 4^{j}$, where $n$ is the size of the input vector $f$. The overall complexity is thus

$$
\sum_{j=0}^{\log _{2}(n) / 2}(4 S)^{j} \frac{n}{4^{j}}= \begin{cases}O\left(n \log _{2}(n)\right) & \text { if } S=1 \\ O\left(n^{1+\log _{2}(S) / 2}\right) & \text { if } S>1\end{cases}
$$

\section{BEST NS WAVELET PACKET DENOISING}

We consider an additive noise model, where a noisy image is obtained as $f=f_{0}+\varepsilon$ where $\varepsilon$ is a Gaussian white noise of variance $\sigma^{2}$.

Thresholding estimator. Following Donoho and Johnstone [8], an estimator $\bar{f}_{\lambda}$ of $f_{0}$ is obtained by hard thresholding at $T>0$ the coefficients of the decomposition of $f$ in a NS wavelet packet basis $\mathcal{B}(\lambda)$

$$
\bar{f}_{\lambda}=\mathcal{W}_{\lambda}^{*}\left(S_{T}\left(\mathcal{W}_{\lambda}(f)\right)\right)
$$

where the hard thresholding operator $S_{T}$ applies to each coefficient the non-linearity $S_{T}(x)=x$ if $|x|>T$ and $S_{T}(x)=0$ otherwise.

Asymptotically minimax optimal estimators are obtained by choosing $T=\sigma \sqrt{2 \log _{e}(P)}$ where $P$ is number of atoms in all NS wavelet packets basis, see [8]. For NS wavelet packet bases, this number is of the order of $P=O\left(n^{1+\log _{2}(S) / 2}\right)$. In practice, good numerical results are obtained with $T \approx 3 \sigma$, and in the numerical results, we select in an oracle manner the $T$ value that minimizes $\left\|f_{0}-\bar{f}_{\lambda}\right\|$.

Best basis for denoising. The best average denoising result is obtained by selecting the basis $\mathcal{B}(\lambda)$ that minimizes the risk $E_{\varepsilon}\left(\left\|f_{0}-\bar{f}_{\lambda}\right\|^{2}\right)$. Following Krim et al. [9], an approximation of the risk is obtained by considering the Lagrangian $L(f, \mathcal{B}(\lambda))$ defined in (3), with the cost function

$$
\Phi(x)=\left\{\begin{array}{l}
x^{2}-\sigma^{2} \text { if }|x| \leqslant T \\
\sigma^{2} \text { if }|x|>T
\end{array}\right.
$$

In the numerical experiments, we set $\rho=T^{2}$ to penalize the complexity of the basis.

The best NS wavelet packet denoising is defined as $\bar{f}_{\lambda^{*}}$ where $\lambda^{\star}$ is the tree that minimizes $L(f, \mathcal{B}(\lambda))$ with the cost (6).

Translation invariant denoising. Once $\lambda^{\star}$ is computed, the denoising quality is greatly improved by using a cycle spinning scheme to reduce thresholding artifacts. For each translation vector $\tau=\left(\tau_{1}, \tau_{2}\right)$, we denote as $\theta_{\tau} f[n]=f[n-\tau]$ the translated image, with periodic boundary conditions. The cycle spinning denoising is obtained with

$$
\tilde{f}_{\lambda^{\star}}=\frac{1}{K^{2}} \sum_{\tau_{1}=0}^{K-1} \sum_{\tau_{2}=0}^{K-1} \theta_{-\tau}\left(\theta_{\tau} f\right)_{\lambda^{\star}}
$$

For the numerical results, we use $K=4$, which increases by $K^{2}=16$ the numerical complexity of the last step of our method.

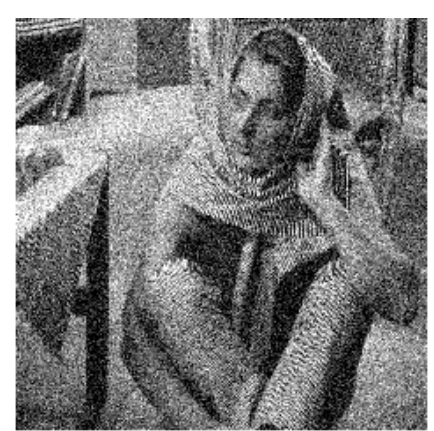

Original PSNR $=19.6 \mathrm{~dB}$

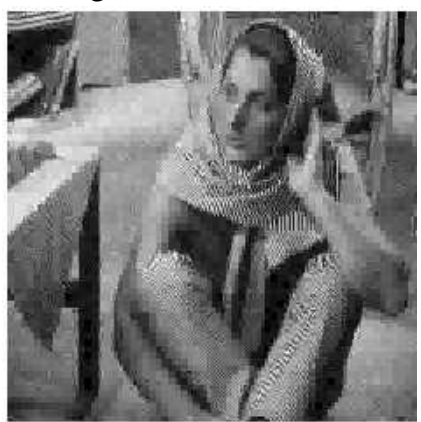

WavePackets PSNR=23.7dB

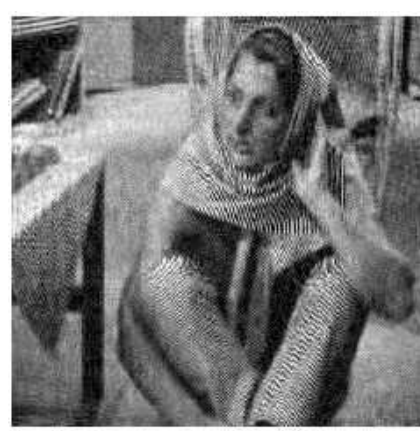

WaveAtoms PSNR=24.4dB

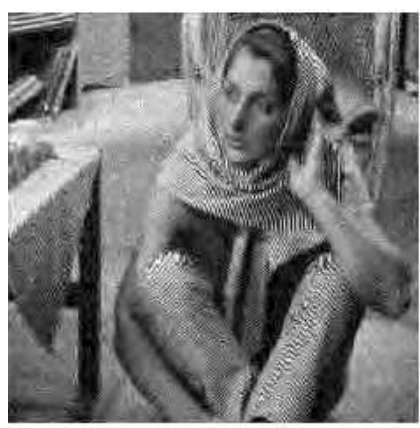

NSWP PSNR=25.4dB
Fig. 2. Comparison of denoising methods on Barbara image.

\section{NUMERICAL RESULTS}

For the numerical experiments, we use $S=6$ Daubechies orthogonal filters $\left\{h_{\ell}\right\}_{\ell=0}^{S-1}$, where $h_{\ell}$ is a filter of length $2 \ell+$ 2.

Figure 2 shows denoising results on a natural image that contains oscillating textures, that is degraded with a noise level $\sigma=0.15\|f\|_{\infty}$. The translation invariant NS wavelet packets improves both over a translation invariant wavelet packet denoising [5] and over the WaveAtoms denoising [4], that are both known for their efficiency to restore oscillating textures.

Figure 3 shows an example of denoising of a fingerprint texture, for $\sigma=.2\|f\|_{\infty}$. Switching from orthogonal NS wavelet denoising to translation invariant denoising leads to a PSNR improvement of $2 \mathrm{~dB}$ on this texture. This shows the importance of this cycle spinning extension. 


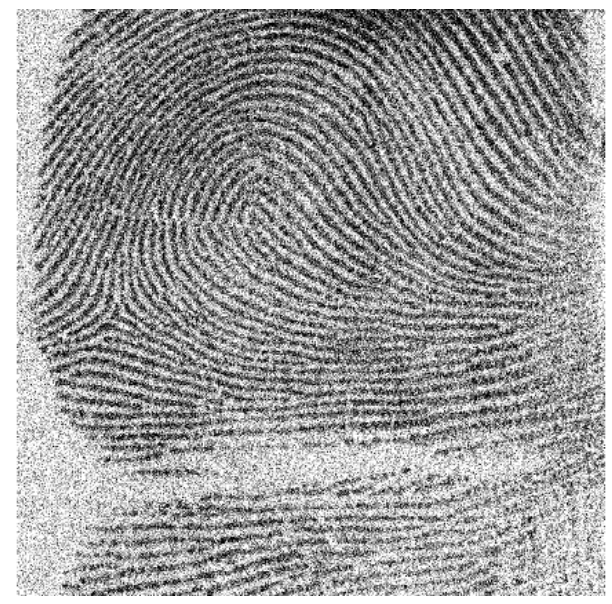

Original PSNR $=18.0 \mathrm{~dB}$

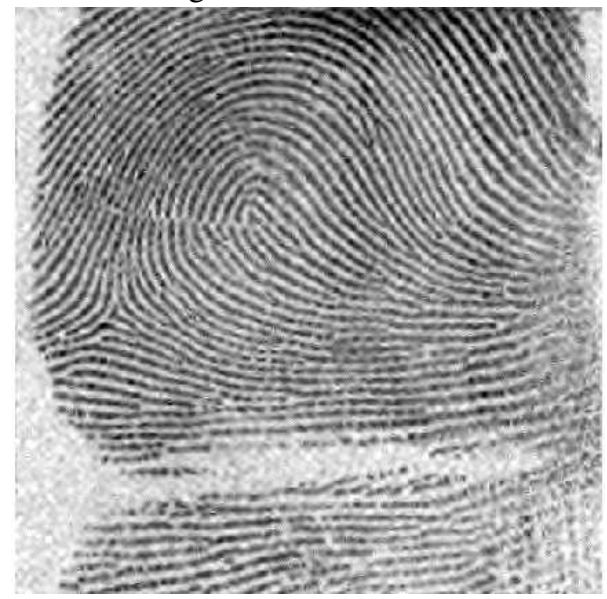

NSWP PSNR $=24.5 \mathrm{~dB}$

Fig. 3. Denoising of a fingerprint texture using the NS wavelet packet algorithm with cycle spinning.

\section{CONCLUSION}

This article has presented a new algorithm to compute a best basis in a non-stationary wavelet packets dictionary. Thresholding the NS wavelet packets coefficients in a best basis computed from a noisy observation performs an adaptive denoising of the image. The translation extension of this thresholding is competitive with the state of the art methods to denoise oscillating textures.

\section{REFERENCES}

[1] S. Mallat, A Wavelet Tour of Signal Processing, Academic Press, San Diego, 1998.

[2] E. Candès and D. Donoho, "New tight frames of curvelets and optimal representations of objects with piecewise $\mathrm{C}^{2}$ singularities," Comm. Pure Appl. Math., vol. 57, no. 2, pp. 219-266, 2004.

[3] F. G. Meyer and R. R. Coifman, "Brushlets: A tool for directional image analysis and image compression," Journal of Appl. and Comput. Harmonic Analysis, vol. 5, pp. 147-187, 1997.

[4] L. Demanet and L. Ying, "Wave atoms and sparsity of oscillatory pat-
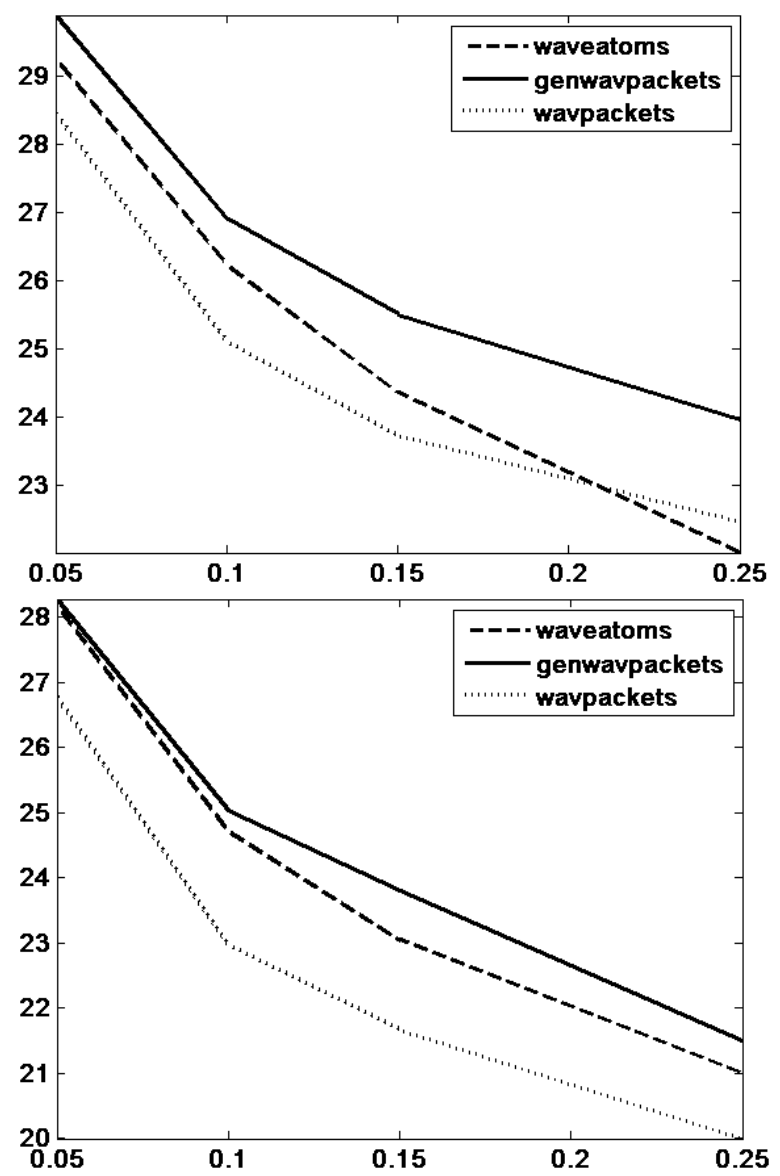

Fig. 4. Curves showing the PSNR (in $\mathrm{dB}$ ) as a function of $\sigma /\|f\|_{\infty}$ for Barbara (top) and Fingerprint (bottom). Solid curves: NS wavelet packets, dashed: WaveAtoms, dotted: wavelet packets.

terns," Applied and Computational Harmonic Analysis, vol. 23, no. 3, pp. 368-387, 2007.

[5] R. R. Coifman and M. V. Wickerhauser, "Entropy-based algorithms for best basis selection," IEEE Transactions on Information Theory, vol. 38, no. 2, pp. 713-718, 1992.

[6] A. Cohen and E. Séré, "Time-frequency localization by non-stationary wavelet packets," in Subband and Wavelet Transforms - Theory and Design, ed. M. T. Smith and A. Akansu, Kluwer Academic Publisher, 1996.

[7] Y. Huang, I. Pollak, M. N. Do, and C. A. Bouman, "Fast search for best representations in multitree dictionaries," IEEE Trans. Image Processing, vol. 15, no. 7, pp. 1779-1793, July 2006.

[8] D. L. Donoho and I. M. Johnstone, "Ideal spatial adaptation via wavelet shrinkage," Biometrika, vol. 81, pp. 425-455, 1994.

[9] H. Krim, D. Tucker, S. Mallat, and D. L. Donoho, "On denoising and best signal representation," IEEE Transactions on Information Theory, vol. 45, no. 7, pp. 2225-2238, 1999. 